\title{
Solution-processed omnidirectional antireflection coatings on amorphous silicon solar cells
}

\author{
Yuehui Wang, ${ }^{1,2, a)}$ Rajesh Tummala, ${ }^{3}$ L. Chen, ${ }^{1}$ L. Qing Guo, ${ }^{4}$ Weidong Zhou, ${ }^{1, a)}$ and \\ Meng Tao ${ }^{1, a)}$ \\ ${ }^{1}$ Department of Electrical Engineering, NanoFAB Center, University of Texas at Arlington, Arlington, \\ Texas 76019-0072, USA \\ ${ }^{2}$ Department of Chemistry and Biology, University of Electronic Science and Technology of China, \\ Zhongshan Institute, Zhongshan 528402, China \\ ${ }^{3}$ Department of Materials Science and Engineering, University of Texas at Arlington, Arlington, \\ Texas 76019, USA \\ ${ }^{4}$ ZT Solar, Inc., 1120 South Freeway, Fort Worth, Texas 76104, USA
}

(Received 11 November 2008; accepted 18 March 2009; published online 18 May 2009)

\begin{abstract}
Solution-processed spherical surface textures are demonstrated on commercial amorphous silicon solar cells. The texture is formed with a monolayer of silica microspheres by convective coating, followed by a spin-on-glass film. It is found that the spherical texture reduces the reflectivity of the cells in the wavelength regime of 400-1200 nm, thus broad spectrum. It is also found that the spherical texture improves the efficiency of the cells at various incident angles from surface normal to at least $60^{\circ}$. The improvement in efficiency increases at larger incident angles to as high as $12 \%$ relative improvement, demonstrating its omnidirectionality. Current-voltage characteristics show that the efficiency improvement with coating is largely due to the increased short circuit current, while the open-circuit voltage remains the same. Therefore the efficiency improvement is attributed to more light coupled into the cells. This omnidirectional surface texture offers an attractive solution for antireflection in both polycrystalline silicon and thin-film solar cells. (C) 2009 American Institute of Physics. [DOI: 10.1063/1.3118499]
\end{abstract}

\section{INTRODUCTION}

All semiconductor materials have high refractive indices in the spectral range from visible to near-infrared, resulting in high Fresnel reflection at the air-semiconductor interface. Therefore, a key issue toward increasing the efficiency of semiconductor-based solar cells is the methods to reduce the interfacial reflection in the wavelength regime of interest to solar cells. ${ }^{1-10}$ A common method is a single-layer quarterwavelength antireflection (AR) coating, such as a silicon nitride film on silicon solar cells or a transparent conducting oxide film on thin-film solar cells. ${ }^{8}$ However, this coating is effective only for a particular wavelength, not broad spectrum. The graded-index structure can be in principle used for broad-spectrum AR, and the thickness and refractive index of each film in the multilayer structure must fall into specific ranges. ${ }^{1,2}$ Surface texturing by anisotropic etching utilizing alkaline solutions such as potassium hydroxide, sodium hydroxide, or tetramethylammonium hydroxide is employed to make pyramidal AR structures on single-crystal Si wafers. ${ }^{3-7}$ For polycrystalline Si and thin-film solar cells, this method does not work because of the various crystallographic orientations of the grains and in some cases the amorphous crystal structure. There is currently no cost-effective method for surface texturing on these solar cells.

Surface texturing by solution deposition has recently been proposed as a cost-effective method for omnidirectional AR (omni-AR) in solar cells. ${ }^{9,10}$ The omni-AR structure is

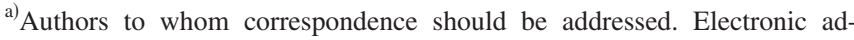
dresses: yhwang@uta.edu,wzhou@uta.edu, and mtao@uta.edu.
}

formed by coating solar cells with a monolayer of microscale spherical dielectric particles, followed by a dielectric film with film thickness of less than the diameter of the spherical dielectric particles. The performance of the omni-AR structure can be controlled by tuning the size, shape, refractive index and packing density of the dielectric particles, and the thickness and the refractive index of the dielectric film. More recently, large-area self-assembled monolayers of silica microspheres have been demonstrated on glass, quartz, and $\mathrm{Si}$ wafers of up to $6 \times 6 \mathrm{in}^{2}$ in size by convective coating. ${ }^{11}$ The ability to obtain large-area uniform monolayers of silica microspheres allows the omni-AR structure to be tested on commercial solar cells. This paper presents a detailed study on the performance of the silica-based omni-AR structure on commercial amorphous Si solar cells.

\section{EXPERIMENTAL}

The suspension of monodispersed silica microspheres with a diameter of $2 \mu \mathrm{m}$ was supplied by MicrospheresNanospheres, a Corpuscular Co. The suspension was used without further purification. The dielectric film was formed by spin-on-glass (SOG), which was supplied by Honeywell with a specified thickness of $0.22 \mu \mathrm{m}$ (Accuglass ${ }^{\circledR} 211$ ). Commercial amorphous Si solar cells from PowerFilm, PowerFilm ${ }^{\circledR}$ S3-37 with a size of $64 \times 37 \mathrm{~mm}^{2}$, were used in the experiment. The commercial cells came encapsulated in plastic. The plastic cover was removed, and the solar cell was bound on a glass wafer to make it flat for deposition of monolayer silica microspheres and SOG. 


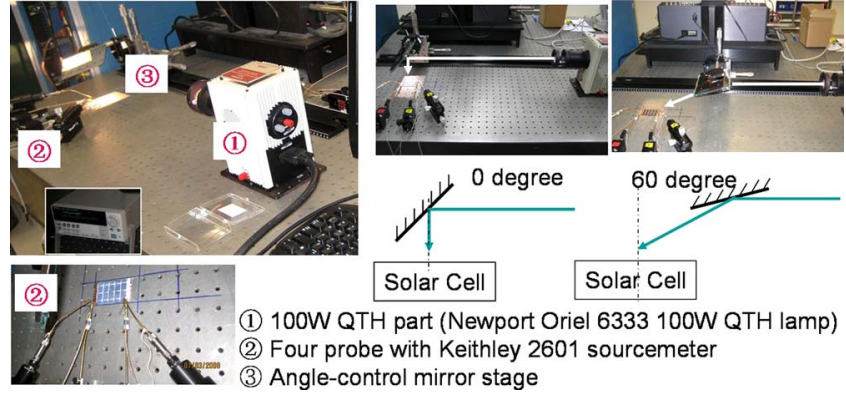

FIG. 1. (Color online) Experimental setup for angle-dependent $I-V$ measurements. Incident angles of $0^{\circ}$ and $60^{\circ}$ are shown as examples.

The formation of uniform monolayers of $2 \mu \mathrm{m}$ silica microspheres by convective coating and the formation of omni-AR structures by spin-coating a SOG film are described in an earlier publication by the authors. ${ }^{11} \mathrm{~A}$ few coating parameters are adjusted to allow the formation of uniform and closely packed monolayers of silica microspheres on the amorphous $\mathrm{Si}$ solar cells, including silica microsphere concentration in the suspension, coating plate speed, dispersed suspension volume, and wedge angle. ${ }^{11}$

The reflectivity of the omni-AR structure is measured under normal incidence using a JASCO V-570 UV/visible spectrophotometer in the spectral range of 400-1200 nm. An integrating sphere is used in the measurement for total reflectance by collecting all the specular and diffusive components of reflection. The microstructure of omni-AR coatings is studied with a Zeiss SUPRA 55 VP scanning electron microscope (SEM). The surface roughness of solar cells before omni-AR is measured with an Alpha Step IQ surface profiler. Solar cells, with and without omni-AR, are characterized by the current-voltage $(I-V)$ measurement. With illumination under roughly Air Mass (AM) 1.5 conditions $\left(\sim 100 \mathrm{~mW} / \mathrm{cm}^{2}\right.$ and $\left.25{ }^{\circ} \mathrm{C}\right)$, the $I-V$ characterization yields fill factor $(\mathrm{FF})$, open-circuit voltage $\left(V_{\mathrm{oc}}\right)$, short circuit current $\left(I_{\mathrm{sc}}\right)$, and efficiency $(\eta)$. From these parameters, the maximum power output $\left(P_{\max }\right)$ and relative enhancement in efficiency are calculated.

It is worth noting that the angle-dependent $I-V$ measurement is crucial for testing the performance of the omni-AR structure on solar cells. This measurement is performed with a home-made setup, as shown in Fig. 1. The incident light is reflected by a mirror onto the solar cell. By moving and rotating the mirror, the incident angle on the solar cell can be controlled from normal $\left(0^{\circ}\right)$ to $75^{\circ}$. The $I-V$ measurement is performed with four probe technique in order to minimize the serial resistance impact.

\section{RESULTS AND DISCUSSION}

\section{A. Structural characteristics of omni-AR coatings}

Shown in Fig. 2 are a photograph of a PowerFilm amorphous Si solar cell [Fig. 2(a)] and a SEM image of the surface morphology of the cell after removal of the plastic cover [Fig. 2(b)]. The surface of the cell consists of a film of indium tin oxide (ITO) and copper leads as electrodes [Fig. 2(a)]. As shown in Fig. 2(b), the morphology of the ITO film is not smooth nor dense but porous with small interconnected

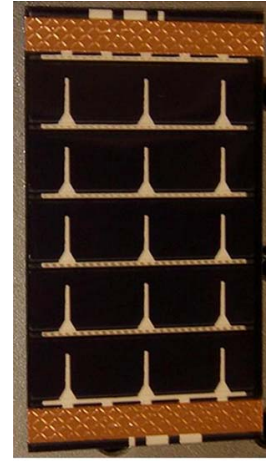

(a)

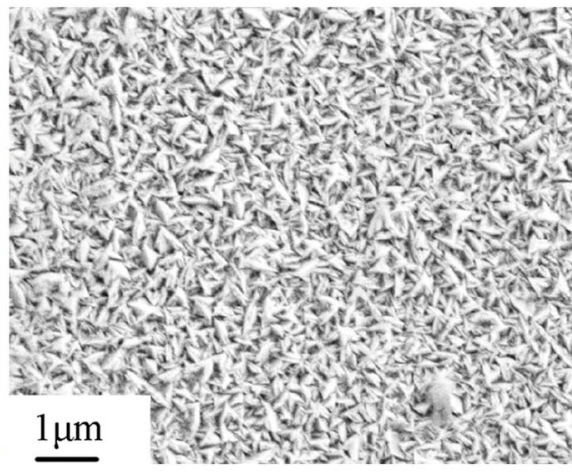

(b)
FIG. 2. (Color online) (a) Photograph of a PowerFilm amorphous Si solar cell and (b) SEM image of the surface morphology of the cell after removal of the plastic cover.

grains. The surface roughness is $0.2-0.3 \mu \mathrm{m}$. The height of the copper leads above the ITO film is $8-10 \mu \mathrm{m}$. These rough surface structures present a challenge for the formation of uniform monolayers of silica microspheres on the entire surface of the cell.

Shown in Fig. 3 are SEM images of a solar cell coated with a monolayer of $2 \mu \mathrm{m}$ silica microspheres [Fig. 3(a)] and followed with a $0.2 \mu \mathrm{m}$ SOG 211 film [Fig. 3(b)]. The insert of Fig. 3(b) is a side view of the omni-AR structure. With proper conditions, relatively uniform silica microsphere monolayers can be obtained on the surface of amorphous $\mathrm{Si}$ solar cells. The microspheres arrange themselves into a hexagonal closely packed structure and look sufficiently uniform, although voids appear in the structure [Fig. 3(a)]. The microspheres are held together by the SOG film [Fig. 3(b)]. No cracks are observed on the entire surface, indicating little stress in the omni-AR structure. It is worth pointing out that the hexagonal closely packed structure is not obtained on the entire surface of the cell. Submonolayers with voids and multilayers of microspheres are observed in some regions. These defects are attributed to the nonuniform distribution of silica microspheres in the meniscus during convective coating and the structural differences on the cell surface. ${ }^{11}$ However, the optical characteristics of the nonperfect omni-AR structure are satisfactory, as will be discussed later.

\section{B. Optical characteristics of omni-AR coatings}

The normal-incidence reflectivity of amorphous $\mathrm{Si}$ solar cells with the omni-AR structure is shown in Fig. 4. For

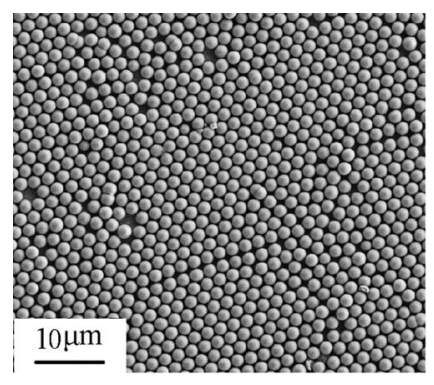

(a)

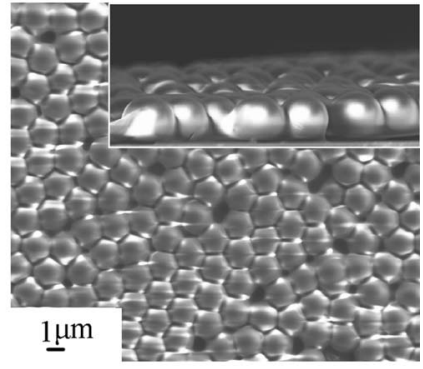

(b)
FIG. 3. (a) SEM image of a solar cell coated with a monolayer of $2 \mu \mathrm{m}$ silica microspheres and (b) followed by a $0.2 \mu \mathrm{m}$ SOG 211 film. The insert in (b) is a side view of the omni-AR structure. 


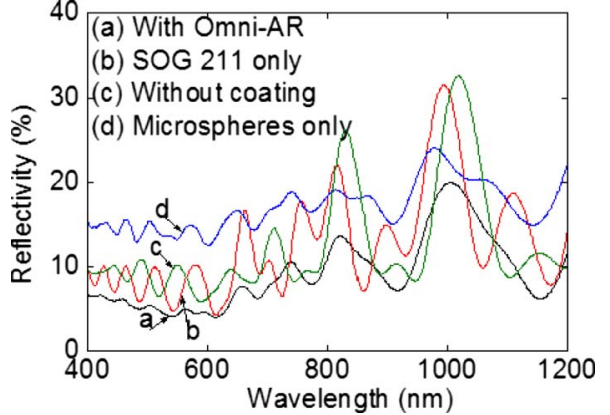

FIG. 4. (Color online) Measured surface-normal reflectance of amorphous Si solar cells (a) without coating, (b) with SOG 211 only, (c) with monolayer silica microspheres only, and (d) with the omni-AR structure.

comparison, the reflectivity of cells without any coating, with SOG 211 only, and with a monolayer of $2 \mu \mathrm{m}$ silica microspheres only is also measured. The omni-AR structure reduces the reflectivity in the spectral range of 400-1200 nm, demonstrating its broad-spectrum nature. It is also noticed that the omni-AR structure shows less spectral dependence than other coatings. The SOG film makes little change in reflectivity as compared to the cell without coating. The microsphere monolayer increases the reflectivity, especially at short wavelengths. This is likely due to reflectivity from multiple surfaces in the microsphere monolayer: the top surface of the microspheres, the bottom surface of the spheres, and the surface of the cell. Therefore, the SOG film not only serves as a glue to hold together the omni-AR structure but also reduces the number of surfaces that cause reflection. ${ }^{9,10}$

The angular and spectral dependences of the transmission of omni-AR structure coated on transparent quartz/glass substrates have been demonstrated in the earlier publication based on home-built integrating sphere setup., ${ }^{9,10}$ Due to the limitation of the experimental setup, we could not measure the reflectivity of amorphous silicon solar cells with omni-AR structure. In here, the simulation results for amorphous silicon solar cells with and without omni-AR structure are carried out to understand the optical performance of the spherical surface textures for AR application on the amorphous solar cells.

The geometrical structure under consideration, along with the key structural parameters, is depicted in Fig. 5. To match the experimental conditions, the structural parameters in the simulation are assumed as following: the substrate material is amorphous silicon and the coating material is ITO thin film with thickness of $h_{2}=50 \mathrm{~nm}$ and index of $n_{\mathrm{ITO}}$ $=1.8$; the omni-AR structure consists of hemispherical silica

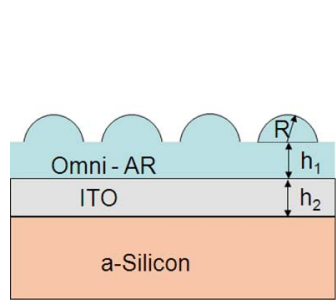

(a)

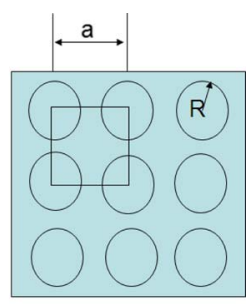

(b)
FIG. 5. (Color online) A hemispherical grating as the basic AR structure for simulation: (a) cross-sectional view; and (b) top view.

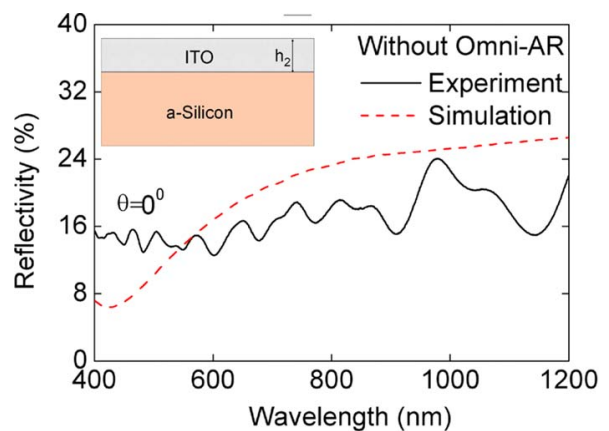

(a)

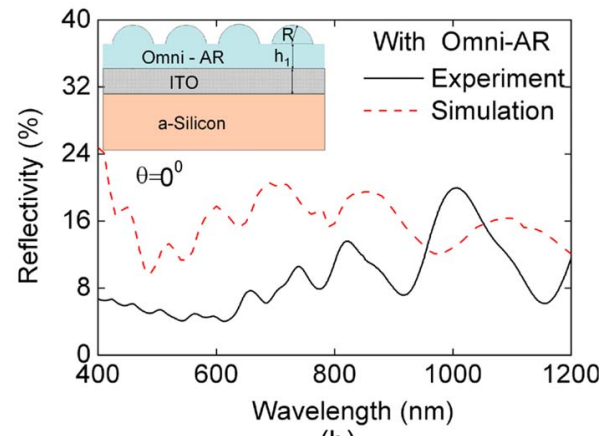

(b)

FIG. 6. (Color online) The measured and simulated reflectivities for $a-\mathrm{Si}$ solar cells (a) without and (b) with omni-AR coatings under surface-normal incident conditions.

microspheres with radius of $R=1 \mu \mathrm{m}\left(n_{\mathrm{SiO}_{2}}=1.46\right)$ and SOG with thickness of $h_{1}=200 \mathrm{~nm} \quad\left(n_{\mathrm{SOG}}=1.46\right)$. The wavelength-dependent refractive index of amorphous silicon $(a-\mathrm{Si})$ is taken from a book. ${ }^{12}$ The average lattice spacing $a=2.5 \mu \mathrm{m}$.

Figure 6 shows the simulated and measured reflectivities of amorphous silicon coated ITO thin film without and with omni-AR structure at surface-normal incident angle. To fit the measured reflectivity for the amorphous silicon solar cell without omni-AR coating, the thickness of the ITO thin film is adjusted during the simulation. We found the best fit is achieved with the ITO thickness of $50 \mathrm{~nm}$, as shown in Fig. 6(a). It is worth noting that the difference in the simulated and measured reflectivities may be largely related to the ITO thin film. As shown in Fig. 2(b), the surface of the ITO film for commercial amorphous $\mathrm{Si}$ cells is not smooth, with small interconnected grains. However, we assumed the ITO thin film to be ideal thin film with smooth surface in the simulation. Based on the same structural parameters, the simulated reflectivity for $a$-Si solar cells with omni-AR coating is shown in Fig. 6(b) along with the measured results. Again, reasonably good agreement can be found.

The simulated reflectivity is shown in Fig. 7 at different incident angles for solar cells without and with omni-AR coating. The improvement in reflectivity with omni-AR structure is relatively small for small incident angles of $0^{\circ}$ and $30^{\circ}$ [Fig. 7(a)]. However, significant improvement in reflectivity can be achieved for large incident angles of $45^{\circ}$ and $60^{\circ}$ [Fig. 6(b)]. This is the most attractive feature of the omni-AR structure, which provides angle independent reflectivity over a wide spectral range. The same result was also 


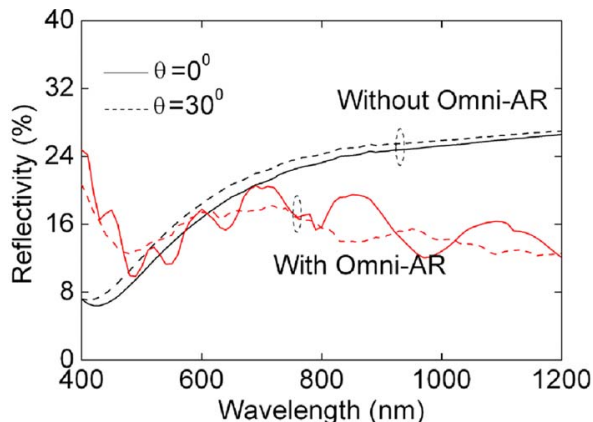

(a)

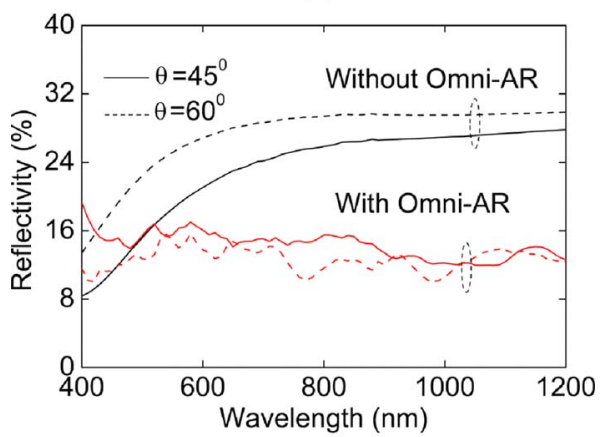

(b)

FIG. 7. (Color online) Simulated reflectivity of amorphous Si cells without and with omni-AR structure at (a) small incident angles $\left(\theta=0^{\circ}\right.$ and $\left.30^{\circ}\right)$ and (b) large incident angles $\left(\theta=45^{\circ}\right.$ and $\left.60^{\circ}\right)$.

obtained in other structures. ${ }^{9,10}$ It is also worth mentioning that further reduction in reflection is feasible with optimized index engineering. ${ }^{10}$

\section{Electrical characteristics of omni-AR coatings}

The effect of omni-AR coating on the solar cell efficiency was examined with the angle-dependent measurement setup for solar cell current-voltage $(I-V)$ measurement. Care was taken to ensure the measurement setup condition is repeatable for various incident angles for solar cells before and after coating. A set of eight solar cells was tested to minimize any statistical variation associated with the test setup. A set of typical measured $I-V$ curves is shown in Fig. 8 for solar cells with different coatings. As shown in Fig. 8(a), when only a monolayer of microspheres was coated on top of the $a$-Si solar cells, the open-circuit voltage $\left(V_{\mathrm{oc}}\right)$ remains largely unchanged, but the short circuit current $\left(I_{\mathrm{sc}}\right)$ reduced for all incident angles measured, indicating a reduction in efficiency, due to the reduced absorption in optical power as a result of increased reflection (Fig. 4).

On the other hand, as shown in Fig. 8(b), short circuit current increased with the omni-AR coating for all incident angles measured. The improvement becomes more prominent at large incident angles. These results agree very well with the simulated reflection results discussed earlier (Fig. 7), demonstrating the omnidirectionality of the spherical coating for improved light absorption with reduced reflection over a wide spectral range and a large range of incident angles.

Figure 9 shows photovoltaic parameters, $I_{\mathrm{sc}}, V_{\mathrm{oc}}$, and $P_{\text {max }}$, as a function of incident angle between surface normal

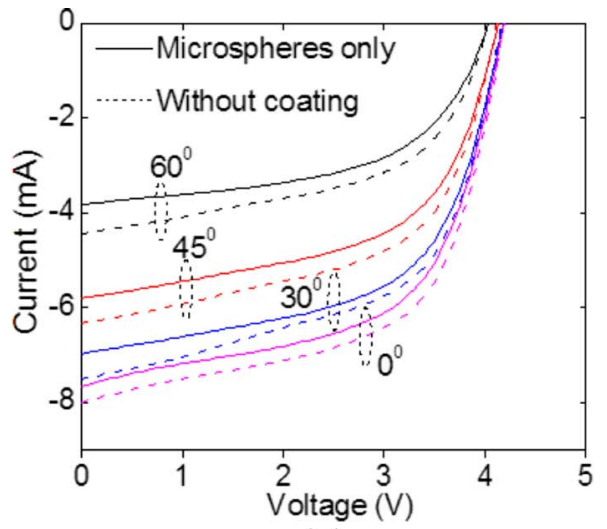

(a)

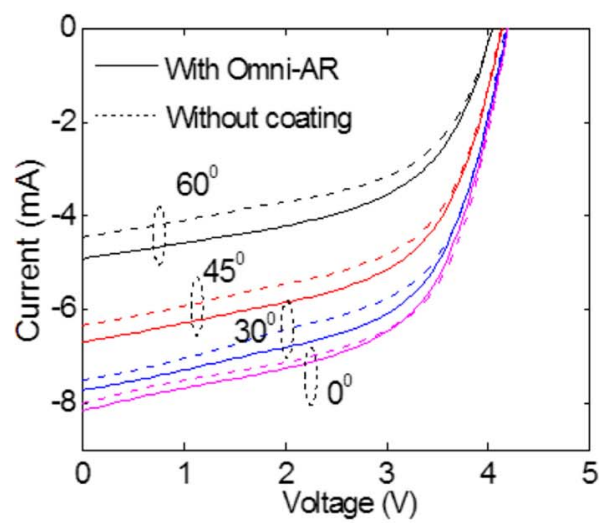

(b)

FIG. 8. (Color online) (a) Measured $I-V$ characteristics of amorphous $\mathrm{Si}$ cells without coating and with monolayer silica microsphere coating only and (b) without coating and with the omni-AR coating structure.

and $60^{\circ}$ for amorphous $\mathrm{Si}$ cells without coating, with a monolayer of silica microspheres only, and with the omni-AR structure. The improvement in $P_{\max }$ with the omni-AR structure comes mainly from larger $I_{\mathrm{sc}}$, while $V_{\mathrm{oc}}$ exhibits negligible change with both the omni-AR structure and a monolayer of silica microspheres. This demonstrates that the cause for increased $P_{\max }$ is the enhanced light coupling into the cell by the omni-AR structure.

\section{Cell performance with omni-AR coatings}

The relative enhancement in efficiency as function of incident angle is shown in Fig. 10 for amorphous Si cells with the omni-AR structure with a monolayer of silica microspheres only and with SOG 211 only. It is clear that only cells with the omni-AR structure show improved efficiency. The improvement becomes more significant at larger incident angles with maximum relative efficiency improvement of $12 \%$ at $60^{\circ}$. Therefore, this omni-AR structure is particularly suitable for fixed-orientation solar cells, such as those installed on rooftops, for increased power output in early mornings and late afternoons. On the other hand, SOG alone or a monolayer of silica microspheres alone reduces the efficiency.

To further confirm the effect of the omni-AR structure, eight amorphous $\mathrm{Si}$ cells were coated with omni-AR for a statistical study. The monolayers of silica microspheres are 


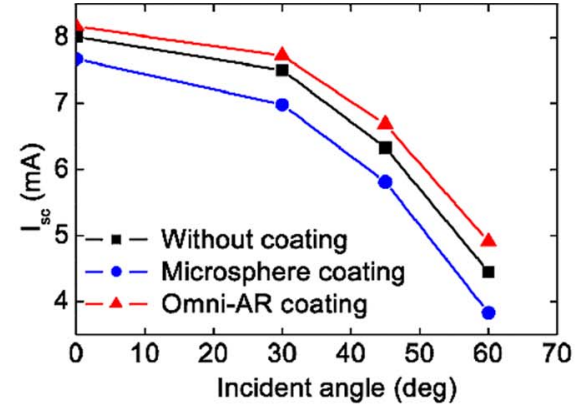

(a)

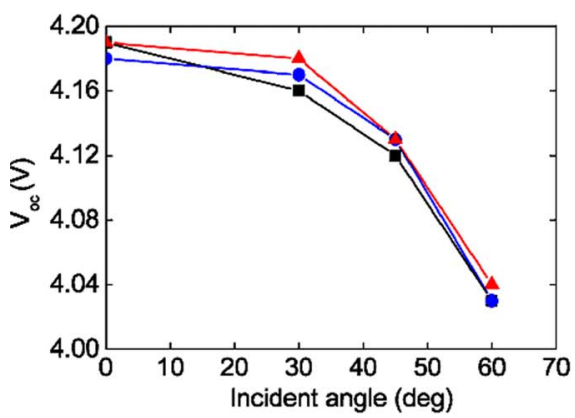

(b)

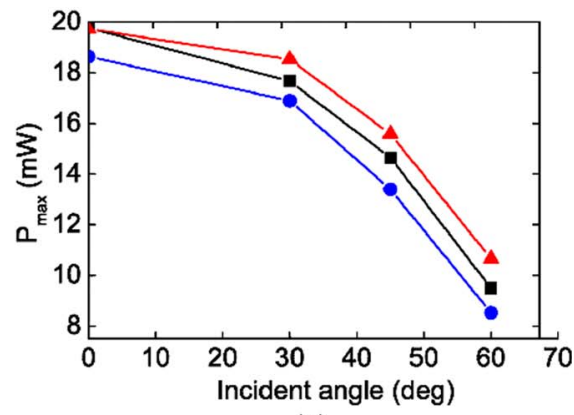

(c)

FIG. 9. (Color online) Photovoltaic parameters as a function of incident angle for amorphous Si cells without coating with monolayer silica microspheres only and with the omni-AR structure. (a) $I_{\text {sc }}$, (b) $V_{\text {oc }}$, and (c) $P_{\max }$.

not perfectly uniform or closely packed, but they do not show multilayers of microspheres or large areas of voids. Figure 11 shows the relative enhancements in efficiency for eight cells measured, with the square symbol indicating the average value. It is certain that the positive effect of the omni-AR structure is statistically meaningful.

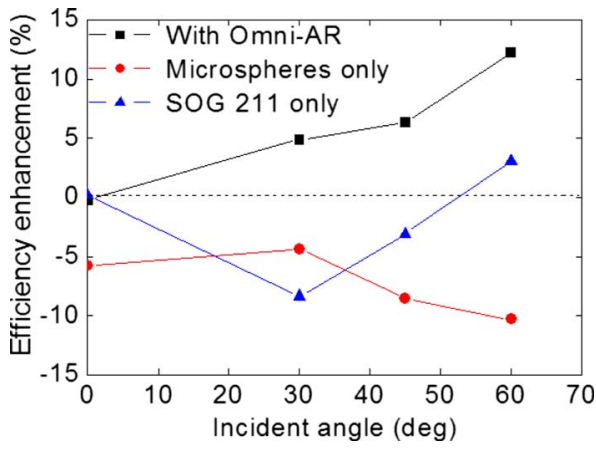

FIG. 10. (Color online) Relative enhancement in efficiency as a function of incident angle for amorphous Si cells with the omni-AR structure with monolayer silica microspheres only and with SOG 211 only.

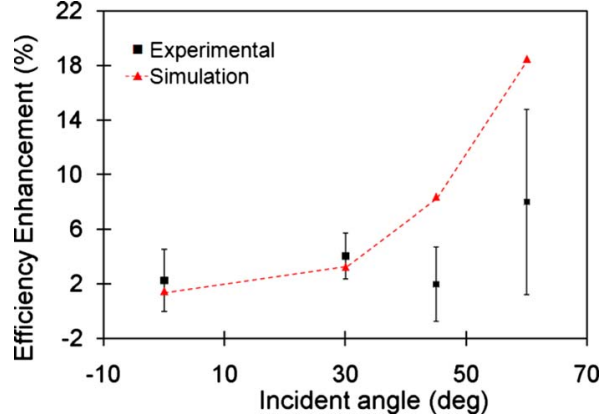

FIG. 11. (Color online) Statistics of relative enhancement in efficiency as a function of incident angle for eight amorphous $\mathrm{Si}$ cells with the omni-AR structure. It also shows the simulated relative enhancement in efficiency with the omni-AR structure.

The solar cell performance can be evaluated theoretically based on the optical characteristics. An outline of the simulation procedure is shown in Fig. 12. The spectral dependent incident photon flux can be calculated based on the measured light source spectra. With the simulated spectral dependent reflection data (e.g., Fig. 7), the total absorbed photon numbers can be calculated. The generated electronhole pairs and the corresponding solar cell short circuit current can then be derived based on the measured internal quantum efficiency data for $a$-Si hydrogenated at different wavelengths ${ }^{13}$ Assuming the coating has no impact on the open-circuit voltage $\left(V_{\text {oc }}\right)$ and FF, the efficiency enhancement can be derived from the ratio of simulated short circuit currents for cells with and without omni-AR coating. The simulated efficiency enhancement result is also shown in Fig. 11. The simulation agrees reasonably well with the measurement. It is worth mentioning that the omni-AR structures obtained in our experiments are not perfectly uniform, which results in the limited improvement in efficiency. With further optimization of the silica microsphere monolayer and use of a different precursor for the dielectric film without impacting the ITO film, more significant improvements in cell performance are expected.

\section{CONCLUSIONS}

Solution-processed inorganic surface textures are demonstrated as a cost-effective AR coating on commercial

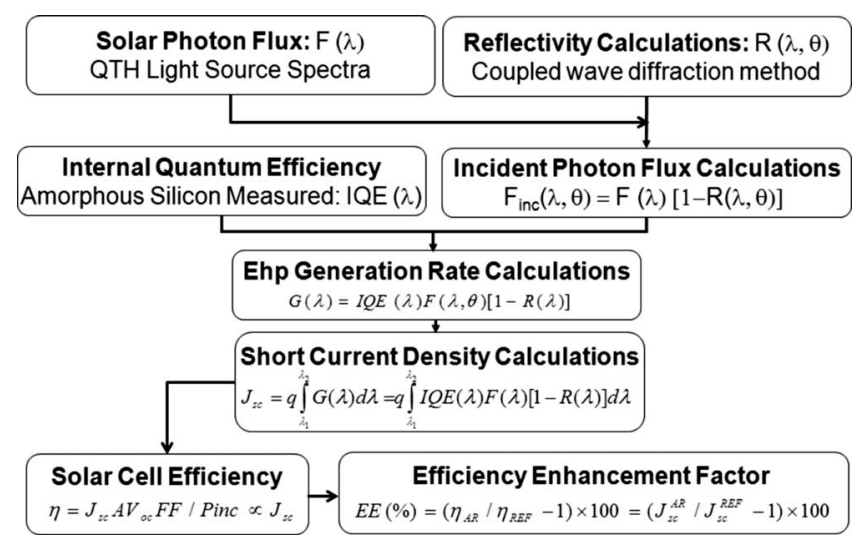

FIG. 12. Simulation procedure for solar cell performance evaluation based on the optical characteristics of solar cell. 
amorphous Si solar cells. The spherical textures are formed with a monolayer of silica microspheres, followed by a SOG film. It is found that the spherical texture reduces the reflectivity of the cells in the wavelength regime of 400-1200 nm, thus broad spectrum. More importantly, the spherical texture improves the efficiency of the cells at various incident angles from surface normal to at least $60^{\circ}$. The improvement in efficiency increases at larger incident angles to as high as $12 \%$ relative improvement, demonstrating its omnidirectionality. Current-voltage characteristics show that the efficiency improvement is largely due to increased short circuit current, while the open-circuit voltage remains the same. Therefore the efficiency improvement is attributed to more light coupled into the cells. This omni-AR structure offers an attractive solution for AR in both polycrystalline $\mathrm{Si}$ solar cells and thin-film solar cells.

\section{ACKNOWLEDGMENTS}

The authors acknowledge financial support for this work from NSF under Grant No. 0740147, Texas Ignition Fund, and AFRL CONTACT Program (Grant No. FA8650-07-2-
5061). The authors also thank H. J. Yang and K. Han for assistance in microstructures and optical characterization.

${ }^{1}$ D. Lee, M. F. Rubner, and R. E. Cohen, Nano Lett. 6, 2305 (2006).

${ }^{2}$ J. Q. Xi, M. F. Schubert, J. K. Kim, E. F. Schubert, M. Chen, S. Y. Lin, W. Liu, and J. A. Smart, Nat. Photonics 1, 176 (2007).

${ }^{3}$ J. Zhao, A. Wang, M. A. Green, and F. Ferrazza, Appl. Phys. Lett. 73, 1991 (1998).

${ }^{4}$ J. D. Hylton, A. R. Burger, and W. C. Sinke, J. Electrochem. Soc. 151, G408 (2004).

${ }^{5}$ É. Vázsonyi, Z. Vértesy, A. Tóth, and J. Szlufcik, J. Micromech. Microeng. 13, 165 (2003).

${ }^{6}$ O. Powell and H. B. Harrison, J. Micromech. Microeng. 11, 217 (2001).

${ }^{7}$ I. Zubel, Sens. Actuators, A 84, 116 (2000).

${ }^{8}$ T. Tsai and Y. Wu, Microelectron. Eng. 83, 536 (2006).

${ }^{9}$ M. Tao, W. Zhou, H. Yang, and L. Chen, Appl. Phys. Lett. 91, 081118 (2007).

${ }^{10}$ W. Zhou, M. Tao, L. Chen, and H. Yang, J. Appl. Phys. 102, 103105 (2007).

${ }^{11}$ Y. Wang, L. Chen, H. Yang, Q. Guo, W. Zhou, and M. Tao, Sol. Energy Mater. Sol. Cells 93, 85 (2009).

${ }^{12}$ E. D. Palik, Handbook of Optical Constants of Solid (Academic, Orlando, 1985).

${ }^{13}$ B. Rech and H. Wagner, Appl. Phys. A: Mater. Sci. Process. 69, 155 (1999). 